\title{
Bulk properties of the van der Waals hard ferromagnet $\mathrm{VI}_{3}$
}

\author{
Suhan Son, ${ }^{1,2}$ Matthew J. Coak, $, 2,3,{ }^{*}$ Nahyun Lee, ${ }^{1}$ Jonghyeon Kim, ${ }^{4}$ Tae Yun Kim, ${ }^{2,5}$ Hayrullo Hamidov, $, 3,6,7$ \\ Hwanbeom Cho, ${ }^{1,2}$ Cheng Liu, ${ }^{3}$ David M. Jarvis, ${ }^{3}$ Philip A. C. Brown, ${ }^{3}$ Jae Hoon Kim ${ }^{4}$ Cheol-Hwan Park, ${ }^{2,5}$ \\ Daniel I. Khomskii, ${ }^{8}$ Siddharth S. Saxena, ${ }^{3,7}$ and Je-Geun Park ${ }^{1,2, *}$ \\ ${ }^{1}$ Center for Correlated Electron Systems, Institute for Basic Science, Seoul 08826, Republic of Korea \\ ${ }^{2}$ Department of Physics and Astronomy, Seoul National University, Seoul 08826, Republic of Korea \\ ${ }^{3}$ Cavendish Laboratory, Cambridge University, J.J. Thomson Ave, Cambridge CB3 OHE, United Kingdom \\ ${ }^{4}$ Department of Physics, Yonsei University, Seoul 03722, Republic of Korea \\ ${ }^{5}$ Center for Theoretical Physics, Seoul National University, Seoul 08826, Republic of Korea \\ ${ }^{6}$ Navoiy Branch of the Academy of Sciences of Uzbekistan, Galaba Avenue, Navoiy 104070, Uzbekistan \\ ${ }^{7}$ National University of Science and Technology MISiS, Leninsky Prospekt 4, Moscow 119049, Russia \\ ${ }^{8}$ II. Physikalisches Institut, Universität zu Köln D-50937 Köln, Germany
}

(Received 13 November 2018; revised manuscript received 28 November 2018; published 7 January 2019)

\begin{abstract}
We present comprehensive measurements of the structural, magnetic, and electronic properties of layered van der Waals ferromagnet $\mathrm{VI}_{3}$ down to low temperatures. Despite belonging to a well-studied family of transitionmetal trihalides, this material has received very little attention. We outline, from high-resolution powder xray diffraction measurements, a corrected room-temperature crystal structure to that previously proposed and uncover a structural transition at $79 \mathrm{~K}$, also seen in the heat capacity. Magnetization measurements confirm $\mathrm{VI}_{3}$ to be a hard ferromagnet $(9.1 \mathrm{kOe}$ coercive field at $2 \mathrm{~K}$ ) with a high degree of anisotropy, and the pressure dependence of the magnetic properties provide evidence for the two-dimensional nature of the magnetic order. Optical and electrical transport measurements show this material to be an insulator with an optical band gap of $0.67 \mathrm{eV}$ - the previous theoretical predictions of $d$-band metallicity then lead us to believe $\mathrm{VI}_{3}$ to be a correlated Mott insulator. Our latest band-structure calculations support this picture and show good agreement with the experimental data. We suggest $\mathrm{VI}_{3}$ to host great potential in the thriving field of low-dimensional magnetism and functional materials, together with opportunities to study and make use of low-dimensional Mott physics.
\end{abstract}

DOI: 10.1103/PhysRevB.99.041402

\section{INTRODUCTION}

Two-dimensional van-der-Waals (vdW) magnetic materials have in recent years become the subject of a wide range of intense research [1]. While a large portion of research into two-dimensional materials has centered on graphene, the addition of magnetism into such a system leads to many interesting fundamental questions and opportunities for device applications [2-6]. Particularly for future spintronics applications, semiconducting or metallic materials which exhibit ferromagnetism down to monolayer thickness are an essential ingredient. This has led to a large volume of recent publications on two-dimensional honeycomb ferromagnet $\mathrm{CrI}_{3}$ [7-12]. $\mathrm{CrI}_{3}$ and $\mathrm{VI}_{3}$ belong to a wider family of $\mathrm{MX}_{3}$ transition metal trihalides with $\mathrm{X}=\mathrm{Cl}, \mathrm{Br}$, I, which were synthesized in the '60s [13,14] but have since seen little interest until recently [15].

$\mathrm{VI}_{3}$ is an insulating two-dimensional ferromagnet with a Curie temperature, $T_{\mathrm{c}}$, given as $55 \mathrm{~K}$ and reported to have the layered crystal structure of $\mathrm{BiI}_{3}$ with space group $R 3$ [16-18]. As shown in a recent review [15], there is very little available information on $\mathrm{VI}_{3}$ other than the structure and the expected

*Corresponding authors: mattcoak@ snu.ac.kr; jgpark10@snu.ac.kr
$S=1$ from the $3 d^{2}$ configuration of the vanadium sites. Calculations using density functional theory, which additionally yield the exchange constants, have suggested $\mathrm{VI}_{3}$ to not only remain ferromagnetic down to a single crystalline layer, but to also exhibit Dirac half-metallicity, of interest for spintronic applications [19].

In these vdW materials, hydrostatic pressure forms an extremely powerful tuning parameter. Given the weak mechanical forces between the crystal planes, the application of pressure will dominantly have the effect of pressing the $a b$ planes together, and gradually and controllably push the system from two- to three-dimensionality. Additionally, firstprinciples calculations have suggested in-plane strain and compression to stabilize both antiferromagnetic phases and spin reorientation in $\mathrm{CrI}_{3}$ [20] and both $\mathrm{VBr}_{3}$ and $\mathrm{VCl}_{3}$ are antiferromagnetic [18].

\section{RESULTS}

\section{A. Crystal structure}

Finding the correct crystal structure is crucial for accurate $a b$ initio calculations of a new material's behavior and for general insight into its properties. The structure of $\mathrm{VI}_{3}$ has not been previously outlined beyond its basic crystallographic family $[13,16,18,21]$. This earlier work assigned $\mathrm{VI}_{3}$ 

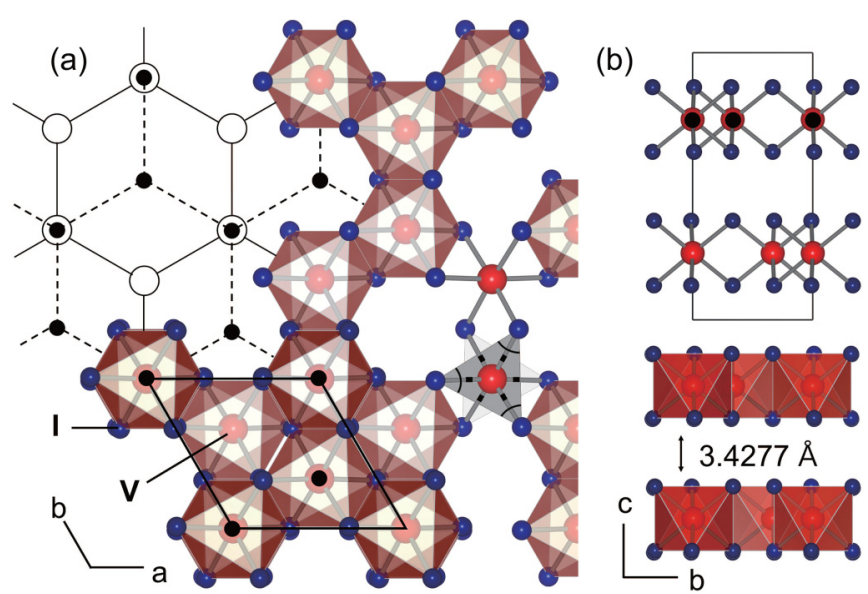

FIG. 1. Views of the room-temperature crystal structure of $\mathrm{VI}_{3}$ along (a) the $c$ axis and (b) the $a$ axis. Additional projections are shown in the SM (Figs. S4 and S5).

as belonging to the $\mathrm{BiI}_{3}$ type $R 3$ structure, but no detailed crystallographic results are available nor any description of temperature dependence. Here we present the crystal structure of $\mathrm{VI}_{3}$ from refined powder $\mathrm{x}$-ray diffraction data and find a transition to an alternative structure at low temperature.

The results show good agreement between the highresolution X-ray diffraction (HR-XRD) measurement and refined data (see Supplementary Material (SM) [22], Figs. S2-S5 and Table S1. The FULLPROF, GSAS-II, and VESTA software suites were employed [23-25]). Contrary to previous reports, we find the room temperature structure to be fitted better with the space group of P3lc than $R 3$. Even though both structures show very similar simulation results, several peaks were missing from the $R 3$ simulations, and peak shapes and ratios are better fit by the P31c (SM, Fig. S3). The P31c structure at room temperature is shown in Fig. 1. The unit cell has an ideal honeycomb bilayer of $\mathrm{V}$ formed of $\left[\mathrm{VI}_{6}\right]^{3-}$ octahedra, separated by a clear van der Waals gap. All the shortest V-V and V-I bonds have the same lengths.

Following the powder-diffraction patterns down to low temperature additionally reveals a phase transition to a new low-temperature structure below $T_{\mathrm{s}}=79.0(5) \mathrm{K}$. Figures 2(a) and 2(b) show heat capacity and details of the x-ray diffraction patterns as a function of temperature. A clear peak at $79 \mathrm{~K}$ in the heat capacity and the peak splitting in the XRD patterns demonstrate the existence of a sharp structural phase transition at this temperature.

As one can see from the long debates about the lowtemperature structure of $\alpha-\mathrm{TiCl}_{3}$ [26], the overlap of many peaks via symmetry breaking at low temperature gives multiple potential solutions to the structure. Among the possible candidates, we obtained the best results for a $C 2 / c$ structure, a subspace group of the room temperature structure. Contrary to $\mathrm{MoCl}_{3}$ [27] and $\alpha-\mathrm{TiCl} 3$ [15] undergoing dimerization of the transition metal ions at low temperature, the $\mathrm{V}$ atoms here stretch in a single direction from their perfect honeycombantidimerization [see Fig. 2(c)]. The short bonding length is 3.92(5) $\AA$ and the long 4.12(11) A. Furthermore, accompanying the antidimerization of $\mathrm{V}$ honeycomb atoms, the $\mathrm{VI}_{6}$ octahedra experience an off-center distortion when the low-

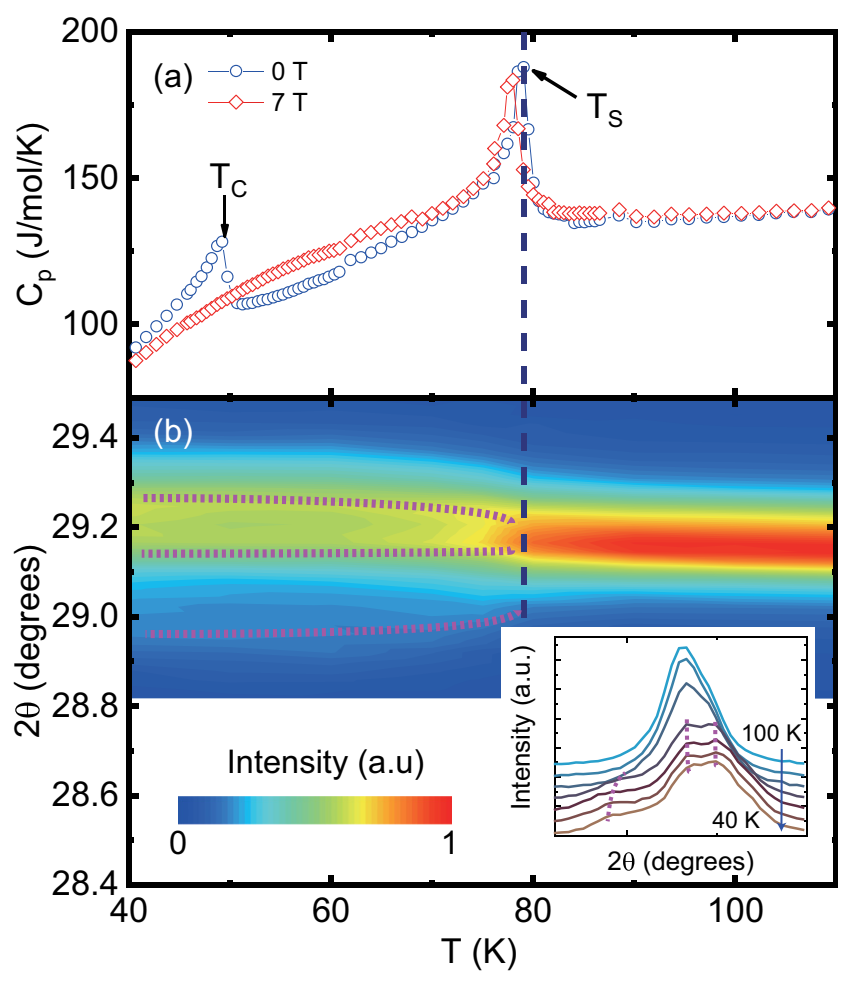

(c)

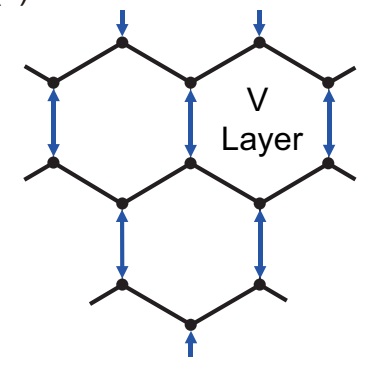

(d)

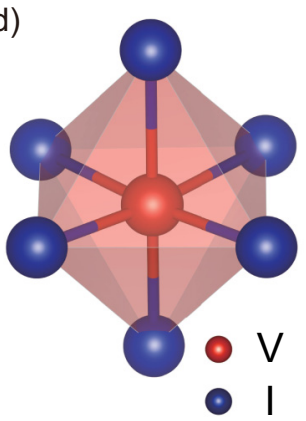

FIG. 2. (a) Heat capacity of a $\mathrm{VI}_{3}$ single crystal, in 0 and $7 \mathrm{~T}$ fields. The structural transition at $T_{\mathrm{s}}=79 \mathrm{~K}$ is unaffected by magnetic field, unlike the ferromagnetic transition at $T_{\mathrm{c}}=50 \mathrm{~K}$. (b) Detail of temperature-dependent X-ray powder diffraction patterns showing peak splitting at $T_{\mathrm{s}}$. (c), (d) Illustrations of the antidimerization of the $\mathrm{V}$ sites and the distortion of the $\mathrm{VI}_{6}$ octahedra in the low-temperature phase.

temperature structure is entered. Figure 2(d) illustrates the structural deformation of the $\mathrm{VI}_{6}$ octahedra at low temperature. Plots of the refinement results and structural parameters at 40 and $300 \mathrm{~K}$ are given in the SM (Figs. S2 and S3, Table S1).

The peak seen in the heat capacity at $50 \mathrm{~K}\left(T_{\mathrm{c}}\right)$ we identify as the ferromagnetic transition from the broadening of peak width in an applied $7 \mathrm{~T}$ of magnetic field. All measurements were reproducible over multiple thermal cycles, which rules out the effect of sample degradation on the results. Like other transition-metal halides with vdW gaps such as $\mathrm{CrI}_{3}$ and $\alpha-\mathrm{RuCl}_{3}, \mathrm{VI}_{3}$ is easily exfoliated by the common Scotch tape method [28] (SM, Fig. S7), placing it as a valuable system for studying two-dimensional physics and applications. 


\section{B. Magnetic properties}

We next explore the magnetic properties of single crystal $\mathrm{VI}_{3}$. The results of measurements are shown in Fig. 3 and additional data given in the SM (Fig. S6). Measurements were carried out with the magnetic field both parallel and perpendicular to the crystallographic $c$ axis, which is easily identified as perpendicular to the $a b$ crystal planes this two-dimensional material forms. Orientation-dependent magnetic susceptibility is shown in Fig. 3(a), measured during warming after cooling to $2 \mathrm{~K}$ with the measurement magnetic field of 100 Oe applied, i.e., field-cooled (FC). Similarly to the previous result of Wilson et al. [18] we find $\mathrm{VI}_{3}$ to be ferromagnetic with $\mathrm{T}_{\mathrm{c}}$ of $50.0(1) \mathrm{K}$, defined from the derivative of the susceptibility.

As shown in Fig. 3(a), there is a strong anisotropy in the susceptibility - magnetic field applied in-plane $(H \perp c)$ leads to magnetization roughly half that found when it is applied out of plane $(H \| c)$. We can conclude that $\mathrm{VI}_{3}$ has Ising-type spins with the easy axis along $c$. A small kink in the magnetic susceptibility as well as a change in slope in the paramagnetic state at $79.3 \mathrm{~K}$ was observed, corresponding to the structural transition temperature $T_{\mathrm{s}}$. A structural-phase transition accompanies similar features in the susceptibility of $\mathrm{CrI}_{3}$ and $\alpha-\mathrm{TiCl}_{3}$ [7,29]. The kink was not seen when the field was applied in-plane, but was clearly present with the field along the easy axis, implying the coupling of the structural phase transition and the magnetism and a link to the magnetic anisotropy.

Zero-FC data are presented in the SM (Fig. S6) - a linear Curie-Weiss fit to these data above $T_{\mathrm{s}}$ allows us to extract an effective moment $\mu_{\text {eff }}=2.08(20) \mu_{B}$. Wilson et al. reported a value of $2.22 \mu_{B}$, which agrees with our fit within error, and our $a b$ initio calculations (SM) give a moment of $2 \mu_{B}$. The extrapolated Curie temperature from the fit is $64.5(5) \mathrm{K}$, which differs from the observed $T_{\mathrm{c}}$ as the structural transition at $T_{\mathrm{s}}$ alters the slope of the susceptibility and destabilizes magnetic order.

The effect of applied hydrostatic pressure on the FC susceptibility in a 1000 Oe field applied along $c$ is shown in Fig. 3(b). Along with the evolution of several bumps in the data, most likely due to domain dynamics, the key result shown in the inset is the behavior of $T_{\mathrm{c}}$. The effect of pressure on a vdW material such as $\mathrm{VI}_{3}$ will be predominately to continuously decrease interlayer spacing, increasing interplanar exchange and tuning toward a three-dimensional system. At pressures up to around $7 \mathrm{kbar}$, however, no change in $T_{\mathrm{c}}$ was observed, but at higher pressures it begins to rapidly increase. We interpret this as evidence of the true two-dimensional nature of the magnetic order in $\mathrm{VI}_{3}$. Bringing the planes closer together has zero effect on stabilizing the magnetic order to higher temperatures-interplanar interactions are unaffected and hence are presumed to be negligible to begin with. Only above 8 kbar does changing interlayer spacing lead to easier formation of magnetic order-at this point, the dimensionality is starting to tune away from two. The only prior pressure study, to our knowledge, on the $\mathrm{MX}_{3}$ materials to date is the work of Yoshida et al. [30], which reports a continuous decrease in the value of $\mathrm{T}_{\mathrm{c}}$ with increasing pressure-the opposite effect to our observations.
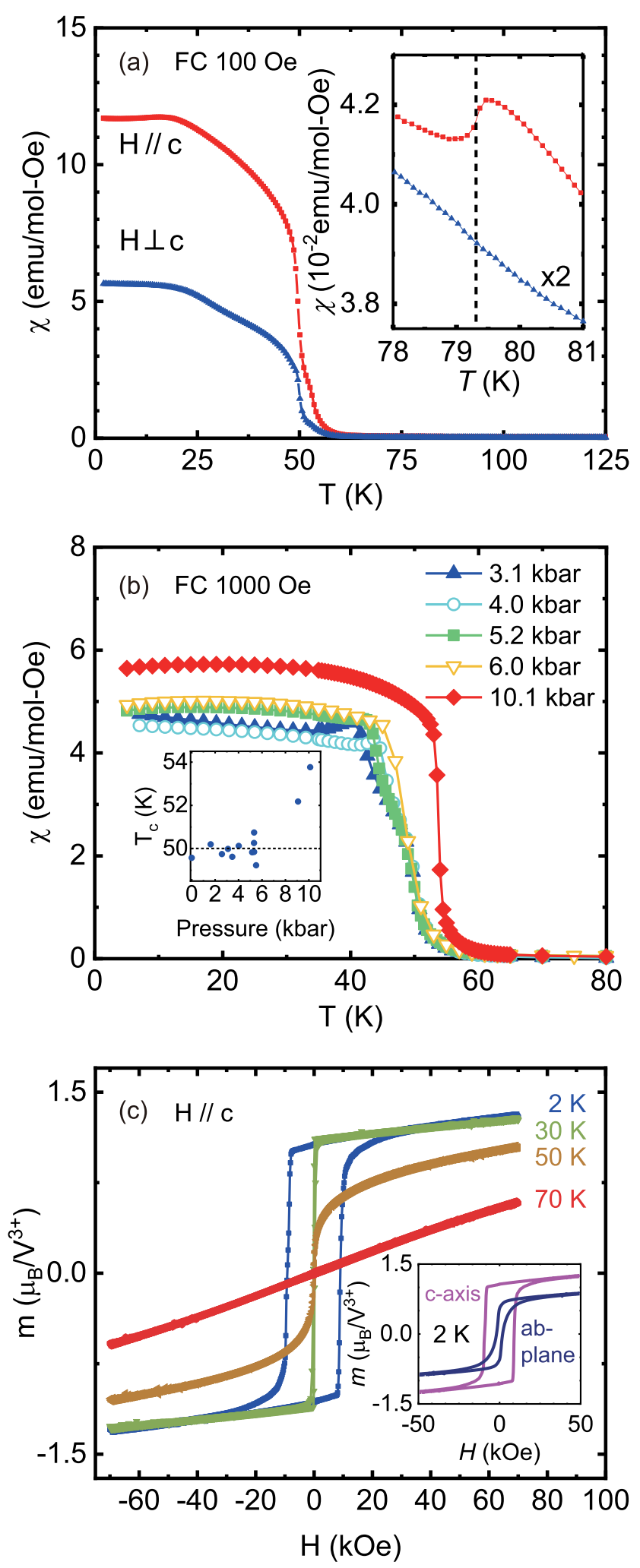

FIG. 3. (a) Field-cooled magnetic susceptibility of $\mathrm{VI}_{3}$ with applied 100 Oe field parallel and perpendicular to the crystallographic $c$ axis. Inset shows detail around $T_{\mathrm{s}}$, where a kink is visible in the data taken with field along $c$. (b) Pressure dependence of the field-cooled data, measured with 1000 Oe along the $c$ direction. Inset shows extracted values of $T_{\mathrm{c}}$. (c) Field-dependent ionic magnetic moment, showing clear ferromagnetic hysteresis loops below $T_{\mathrm{c}}$-inset shows the orientation dependence at $2 \mathrm{~K}$. 
Characteristic ferromagnetic hysteresis loops of the magnetic moment with applied field along the $c$ axis are shown in Fig. 3(c) for several temperatures (more in the SM). As temperature is increased, the coercive field is exponentially decreased (SM, Fig. S6) and above $T_{\mathrm{c}}$ the hysteresis loops close and the system reverts to paramagnetic linear behavior. At the maximum applied field of $70 \mathrm{kOe}(7 \mathrm{~T})$ and at $2 \mathrm{~K}$, the magnetic moment was found to be 1.3(1) $\mu_{B} /$ f.u., which is smaller than the expected saturated moment of a $\mathrm{V}^{3+}$ ion, $g S=2 \mu_{B} /$ f.u. The moment is not fully saturated at $70 \mathrm{kOe}$, but clearly cannot reach this predicted value. The reason for this disagreement is not currently clear.

The orientation dependence of the magnetization is illustrated in the inset of Fig. 3(c). The field-in-plane curves show coercivity of $1.8 \mathrm{kOe}$, smaller than that of out-of-plane $(9.1 \mathrm{kOe})$, which again suggests the easy axis to lie along the $c$ axis, from the Stoner-Wohlfarth model [31]. This simple model, it should be noted, while offering valuable insight into the overall behavior strictly applies only to a singledomain crystal-more complex treatments [32] may prove more suitable for these data going forward. One thing to note here is its huge coercive field at $2 \mathrm{~K}-9.1 \mathrm{kOe}$, in contrast to other vdW ferromagnets such as $\mathrm{CrBr}_{3}$ [33], $\mathrm{CrI}_{3}$ [7], $\mathrm{Fe}_{3} \mathrm{GeTe}_{2}$ [34], and $\mathrm{Cr}_{2} \mathrm{Ge}_{2} \mathrm{Te}_{6}$ [35]. To our knowledge, all other such ferromagnets are soft, contrary to this hard vdW ferromagnet $\mathrm{VI}_{3}$. Understanding the physical origin of the hard-ferromagnetic behavior and the comparison of otherwise similar systems $\mathrm{VI}_{3}$ and $\mathrm{CrI}_{3}$ forms a rich opportunity for low-dimensional magnetism going forward and may help in designing future magnetic materials for specific applications. Interestingly, Chang et al. [36] reported in their comparison of $\mathrm{V}$ - and $\mathrm{Cr}$-doped $\mathrm{Sb}_{2} \mathrm{Te}_{3}$ that the $\mathrm{V}$-doped samples show much higher coercive fields than the $\mathrm{Cr}$-doped systems. The Stoner-Wohlfarth model describes that a small saturated moment, $M_{S}$, and/or a large total magnetic anisotropy $K$ lead to larger coercive fields. The smaller saturated moment in $\mathrm{V}^{3+}$ driven by the smaller number of $d$-orbital spin and the larger anisotropy coming from the partially filled $t_{2 g} d$-band of the $\mathrm{V}^{3+}$ ion, unlike the fully filled $\mathrm{Cr}^{3+}$ orbitals, could contribute the large anisotropy, and, in turn, coercive field.

\section{Electronic properties}

To investigate the electronic properties, we measured the band gap by optical transmittance and the temperature dependence of the bulk resistivity. Figure 4(a) shows the transmittance at room temperature as a function of incident photon energy, from which we can extract the optical band gap. The dependence of the absorption coefficient $\alpha$ on incident photon energy is given by the expression $\alpha E \propto\left(E-E_{g}\right)^{m}$, where $E=h v$ is the incident photon energy, $E_{g}$ is the optical band gap, and for a direct allowed transition the exponent can be taken as $m=1 / 2$. For $E<E_{g}$, the material cannot absorb photons so the band gap is found from extrapolating $(\alpha h v)^{2}$ vs $h v$ to zero (inset). The obtained direct optical band-gap value for $\mathrm{VI}_{3}$ was $0.67(1) \mathrm{eV}$.

The temperature dependence of the in-plane resistivity, $\rho$, of a bulk single crystal of $\mathrm{VI}_{3}$ is plotted in Fig. 4(b) from room temperature down to the point where the resistance becomes too high to measure on our setup. The increasing resistivity as
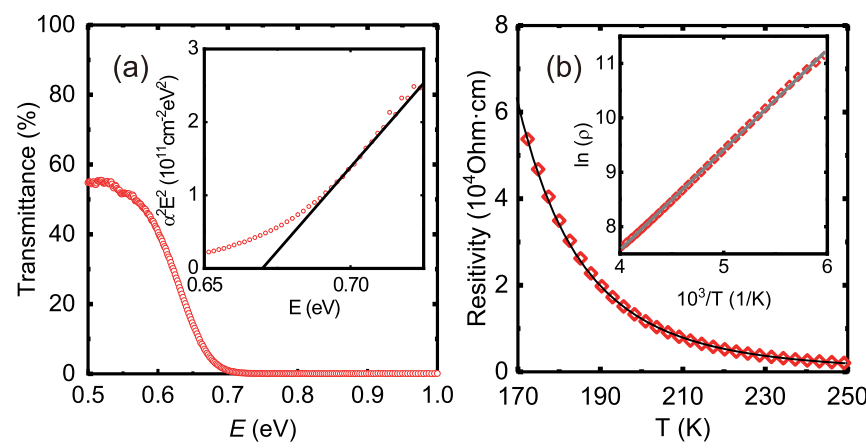

FIG. 4. (a) Optical transmittance of $\mathrm{VI}_{3}$ at room temperature with inset showing the accompanying Tauc plot and linear fit to extract the optical band gap. (b) Resistivity, with good agreement to the Arrhenius insulating temperature dependence shown in the inset.

temperature was lowered shows clear insulating behavior. The resistivity can be fitted well by an Arrhenius type exponential function: $\rho \propto e^{E_{a} / k_{B} T}$, where $E_{a}$ is the activation energy and $k_{B}$ Boltzmann's constant. The inset illustrates the standard $\ln (\rho)$ vs $1 / T$ plot - the straight line proves good agreement to Arrhenius-type thermally activated transport. The extracted activation energy $E_{a}$ was $0.16 \mathrm{eV}$, giving an electrical band gap of $0.32 \mathrm{eV}$ - significantly smaller than the measured optical band gap. The reason for this (common) mismatch is not immediately clear in this material; a potential explanation is the presence of some form of impurity band within the gap or denaturing of the surface contact-iodine deficiency or the effect of exposure to oxygen or moisture may be possible candidates.

Sister compound $\mathrm{CrI}_{3}$ is a Mott insulator [7], with all the rich electronic correlation that implies. Previous results from $a b$ initio calculations [19] predict $\mathrm{VI}_{3}$ to be metallic, rather than the observed insulating behavior-giving a hint that $\mathrm{VI}_{3}$ is also a Mott-insulating system. Our band structure calculations (SM, Fig. S1) implemented in the Quantum ESPRESSO package [37-42], which use our updated crystal structure parameters and include the effects of a Hubbard $U$, suggest a ground state that is indeed Mott insulating, rather than metallic, with a $\sim 1 \mathrm{eV}$ band gap in good agreement with observed data. Reducing the on-site Coulomb interaction leads to half metallicity in our calculations.

\section{DISCUSSION}

We have presented an overview of the basic properties of near-unexplored van der Waals ferromagnet $\mathrm{VI}_{3}$. We suggest an updated crystal structure-crucial for accurate $a b$ initio calculations - and a transition into a distorted alternative structure at low temperature. A key result is a coercive field far higher than in any other vdW ferromagnet system, setting $\mathrm{VI}_{3}$ apart as a hard $\mathrm{vdW}$ ferromagnet and raising several questions worthy of further exploration. Optical and electrical transport measurements show this material to be an insulator with an optical band gap of $0.67 \mathrm{eV}$. In contrast to previously published studies, our band-structure calculations yield an insulating ferromagnetic ground state when an on-site Coulomb interaction is included, leading us to believe $\mathrm{VI}_{3}$ to be a correlated Mott insulator. This evidence of Mott physics evokes potential for band-gap tuning and the emergence of 
exotic states due to strong electron correlations in this $2 \mathrm{D}$ ferromagnetic system.

\section{ACKNOWLEDGMENTS}

The authors would like to thank Sanghyun Lee, S. E. Dutton, Inho Hwang, and Y. Noda for their generous help and discussions. We would also like to acknowledge support from Jesus College of the University of Cambridge, IHT Kazatomprom, and the CHT Uzbekistan Programme.
The work was carried out with financial support from the Ministry of Education and Science of the Russian Federation in the framework of Increase Competitiveness Program of NUST MISiS (No. K2-2017-024). The work of D.Kh. was supported by the German research Project No. SFB 1238 and by Köln University via the German Excellence Initiative. This work was supported by the van der Waals Materials Research Center NRF-2017R1A5A1014862 and the Institute for Basic Science of the Republic of Korea (Grant No. IBS-R009-G1).
[1] P. Ajayan, P. Kim, and K. Banerjee, Phys. Today 69(9), 38 (2016).

[2] J.-G. Park, J. Phys.: Condens. Matter 28, 301001 (2016).

[3] C. Kuo, M. Neumann, K. Balamurugan, H. Park, S. Kang, H. Shiu, J. Kang, B. Hong, M. Han, T. Noh, and J.-G. Park, Sci. Rep. 6, 20904 (2016).

[4] Y. Zhou, H. Lu, X. Zu, and F. Gao, Sci. Rep. 6, 19407 (2016).

[5] N. Samarth, Nature 546, 216 (2017).

[6] K. Burch, D. Mandrus, and J.-G. Park, Nature 563, 47 (2018).

[7] M. McGuire, H. Dixit, V. Cooper, and B. Sales, Chem. Mater. 27, 612 (2015).

[8] W.-B. Zhang, Q. Qu, P. Zhu, and C.-H. Lam, J. Mater. Chem. C 3, 12457 (2015).

[9] H. Wang, F. Fan, S. Zhu, and H. Wu, Europhys. Lett. 114, 47001 (2016).

[10] B. Huang, G. Clark, E. Navarro-Moratalla, D. Klein, R. Cheng, K. Seyler, D. Zhong, E. Schmidgall, M. A. McGuire, D. H. Cobden, W. Yao, D. Xiao, P. Jarillo-Herrero, and X. Xu, Nature 546, 270 (2017).

[11] J. L. Lado and J. Fernández-Rossier, 2D Mater. 4, 035002 (2017).

[12] D. R. Klein, D. MacNeill, J. L. Lado, D. Soriano, E. NavarroMoratalla, K. Watanabe, T. Taniguchi, S. Manni, P. Canfield, J. Fernández-Rossier, and P. Jarillo-Herrero, Science 360, 1218 (2018).

[13] D. Juza, D. Giegling, and H. Schäfer, Z. Anorg. Allg. Chem. 366, 121 (1969).

[14] J. Dillon and C. Olson, J. Appl. Phys. 36, 1259 (1965).

[15] M. McGuire, Crystals 7, 121 (2017).

[16] J. Trotter and T. Zobel, Z. Kristallogr. 123, 67 (1966).

[17] L. L. Handy and N. W. Gregory, J. Am. Chem. Soc. 72, 5049 (1950).

[18] J. Wilson, C. Maule, P. Strange, and J. Tothill, J. Phys. C 20, 4159 (1987).

[19] J. He, S. Ma, P. Lyu, and P. Nachtigall, J. Mater. Chem. C 4, 2518 (2016).

[20] F. Zheng, J. Zhao, Z. Liu, M. Li, M. Zhou, S. Zhang, and P. Zhang, arXiv:1709.05472.

[21] K. Berry, R. Smardzewski, and R. McCarley, Inorg. Chem. 8, 1994 (1969).

[22] See Supplemental Material at http://link.aps.org/supplemental/ 10.1103/PhysRevB.99.041402 for details of structural refinements and results, experimental methods, and additional plots.

[23] J. Rodríguez-Carvajal, Physica B (Amsterdam) 192, 55 (1993).

[24] B. H. Toby and R. B. Von Dreele, J. Appl. Crystallogr. 46, 544 (2013).
[25] K. Momma and F. Izumi, J. Appl. Crystallogr. 44, 1272 (2011).

[26] G. Natta, P. Corradini, and G. Allegra, J. Polym. Sci. 51, 399 (1961).

[27] H. Hillebrecht, P. Schmidt, H. Rotter, G. Thiele, P. Zönnchen, H. Bengel, H.-J. Cantow, S. Magonov, and M.-H. Whangbo, J. Alloys Compd. 246, 70 (1997).

[28] K. S. Novoselov, A. Geim, S. V. Morozov, D. Jiang, Y. Zhang, S. V. Dubonos, I. V. Grigrieva, and A. A. Firsov, Science 306, 666 (2004)

[29] K. Tsutsumi, H. Okamoto, C. Hama, and Y. Ishihara, J. Magn. Magn. Mater. 90-91, 181 (1990).

[30] H. Yoshida, J. Chiba, T. Kaneko, Y. Fujimori, and S. Abe, Physica B (Amsterdam) 237-238, 525 (1997).

[31] E. Stoner and E. Wohlfarth, Philos. Trans. R. Soc. London A 240, 599 (1948).

[32] S. M. Ryabchenko and V. M. Kalita, J. Exp. Theor. Phys. 118, 284 (2014).

[33] N. Richter, D. Weber, F. Martin, N. Singh, U. Schwingenschlögl, B. V. Lotsch, and M. Kläui, Phys. Rev. Materials 2, 024004 (2018).

[34] N. León-Brito, E. D. Bauer, F. Ronning, J. D. Thompson, and R. Movshovich, J. Appl. Phys. 120, 083903 (2016).

[35] C. Gong, L. Li, Z. Li, H. Ji, A. Stern, Y. Xia, T. Cao, W. Bao, C. Wang, Y. Wang, Z. Qiu, R. Cava, S. Louie, J. Xia, and X. Zhang, Nature 546, 265 (2017).

[36] C.-Z. Chang, W. Zhao, D. Y. Kim, H. Zhang, B. A. Assaf, D. Heiman, S.-C. Zhang, C. Liu, M. H. W. Chan, and J. S. Moodera, Nat. Mater. 14, 473 (2015).

[37] P. Giannozzi, S. Baroni, N. Bonini, M. Calandra, R. Car, C. Cavazzoni, D. Ceresoli, G.L. Chiarotti, M. Cococcioni, I. Dabo, A. Dal Corso, S. de Gironcoli, S. Fabris, G. Fratesi, R. Gebauer, U. Gerstmann, C. Gougoussis, A. Kokalj, M. Lazzeri, L. Martin-Samos, N. Marzari, F. Mauri, R. Mazzarello, S. Paolini, A. Pasquarello, L. Paulatto, C. Sbraccia, S. Scandolo, G. Sclauzero, A. P. Seitsonen, A. Smogunov, P. Umari and R. M. Wentzcovitch, J. Phys.: Condens. Matter 21, 395502 (2009).

[38] S. L. Dudarev, G. A. Botton, S. Y. Savrasov, C. J. Humphreys and A. P. Sutton, Phys. Rev. B 57, 1505 (1998).

[39] D. R. Hamann, Phys. Rev. B 88, 085117 (2013); 95, 239906(E) (2017).

[40] M. Schlipf and F. Gygi, Comput. Phys. Commun. 196, 36 (2015).

[41] J. P. Perdew, K. Burke and M. Ernzerhof, Phys. Rev. Lett. 77, 3865 (1996).

[42] H. J. Monkhorst and J. D. Pack, Phys. Rev. B 13, 5188 (1976). 